\title{
Polygons and iteratively regularizing affine transformations
}

\author{
Otto Roeschel $^{1}$
}

Received: 30 May 2016 / Accepted: 5 September 2016 / Published online: 19 September 2016 (C) The Author(s) 2016. This article is published with open access at Springerlink.com

\begin{abstract}
We start with a generic planar $n$-gon $Q_{0}$ with veritices $q_{j, 0}(j=0, \ldots, n-$ 1 ) and fixed reals $u, v, w \in \mathbb{R}$ with $u+v+w=1$. We iteratively define $n$-gons $Q_{k}$ of generation $k \in \mathbb{N}$ with vertices $q_{j, k}(j=0, \ldots, n-1)$ via $q_{j, k}:=u q_{j, k-1}+$ $v q_{j+1, k-1}+w q_{j+2, k-1}$. We are able to show that this affine iteration process for general input data generally regularizes the polygons in the following sense: There is a series of affine mappings $\beta_{k}$ such that the sums $\Delta_{k}$ of the squared distances between the vertices of $\beta_{k}\left(Q_{k}\right)$ and the respective vertices of a given regular prototype polygon $P$ form a null series for $k \longrightarrow \infty$.
\end{abstract}

Keywords Affine Iterations · Affine Regularization · Regular $n$-gons

Mathematics Subject Classification 51 N10 $\cdot 51$ N20

\section{Introduction}

Ziv (2002) and Donisi et al. (2016) studied a geometric iteration process starting with a generic planar polygon $Q_{0}$ with $n>2$ vertices $\left\{q_{0,0}, \ldots, q_{n-1,0}\right\}\left(q_{j, 0} \in \mathbb{C}\right)$. This process places homothetic copies of a given fixed triangle $X Y Z$ on the sides of $Q_{0}$. This way the third point $Z$ of $X Y Z$ gets them the points of a next generation polygon $Q_{1}$. Reapplying this process over and over again with the same triangle $X Y Z$ creates a series of generations $Q_{k}$. Surprisingly, this iteration process, in general, has a regularizing effect on the polygon: The generation $Q_{k}$ approaches the shape of a

$凶$ Otto Roeschel

roeschel@tugraz.at

1 Institute of Geometry, NAWI Graz, Graz University of Technology, Kopernikusgasse 24, 8010 Graz, Austria 
regular $n$-gon-see Donisi et al. (2016) and Ziv (2002). A general cyclic iteration process was introduced by Schoenberg (1950). He reports conditions and results on the existence of a limit point of these iterations. We will show that a regularizing effect generally shows up for affine iteration processes of this type. In order to prove this surprising property we set up an appropriate definition of 'affine regularization'.

\section{The affine iteration process}

We use complex numbers $z \in \mathbb{C}$ with $z=x+i y(x, y \in \mathbb{R})$ to describe points of the Euclidean plane $\mathbb{R}^{2}$ and start with a generic planar polygon $Q_{0}$ with $n>2$ vertices $\left\{q_{0,0}, q_{1,0}, \ldots, q_{n-1,0}\right\}$ with $q_{j, 0} \in \mathbb{C}$. Additionally, we choose a reference triangle $a b c$ and a reference point $z^{*}:=u a+v b+w c$ with fixed barycentrics $(u, v, w) \in \mathbb{R}^{3}$ $(u+v+w=1)$ with respect to $a b c$.

We now define the affine mappings $\alpha_{j, 1}$ from the ordered vertex set $a b c$ of the reference triangle to ordered triples of consecutive vertices $q_{j, 0}, q_{j+1,0}, q_{j+2,0}$ of $Q_{0}(j \in \mathbb{N} ; j \bmod n)$. The point $z^{*}$ is mapped into points $\alpha_{j, 1}\left(z^{*}\right):=q_{j, 1}=$ $u q_{j, 0}+v q_{j+1,0}+w q_{j+2,0}(j \in \mathbb{N}, j \bmod n)$. These points form vertices of the generation 1 polygon $Q_{1}$. Iterations of this process yield affine mappings $\alpha_{j, k}$ and points

$$
\begin{aligned}
\alpha_{j, k}\left(z^{*}\right): z^{*} & =u a+v b+w c \longrightarrow \alpha_{j, k}\left(z^{*}\right):=q_{j, k} \text { with } \\
q_{j, k} & =u q_{j, k-1}+v q_{j+1, k-1}+w q_{j+2, k-1}(k \in \mathbb{N}-\{0\})
\end{aligned}
$$

$(j \in \mathbb{N}, j \bmod n)$ forming a series of polygons $Q_{k}:=\left(q_{0, k}, q_{1, k}, \ldots, q_{n-1, k}\right)$ called the $k$ th generation polygons. This procedure generalizes the geometric iteration process presented and discussed in Radcliffe (2016). In the sequel $Q_{k}$ also denotes the vectors $Q_{k}:=\left(q_{0, k}, \ldots, q_{n-1, k}\right)^{t} \in \mathbb{C}^{n}$. Figure 1 shows an example of the first iteration of procedure (1) for $n=5$.

As we are interested in affine properties of the iteration process we describe affine mappings of the plane $\mathbb{R}^{2}$ by complex numbers: With $z=x+i y(x, y \in \mathbb{R}$, let the conjugate number to $z$ be named $\bar{z}:=x-i y)$ an affine mapping $\beta: \mathbb{R}^{2} \longrightarrow \mathbb{R}^{2}$ is given by $(x, y) \longrightarrow \beta(x, y)=\left(x^{*}, y^{*}\right):=\left(d_{1}+a_{1} x-b_{2} y, d_{2}+a_{2} x+b_{1} y\right)$ with $d_{1}, d_{2}, a_{1}, a_{2}, b_{1}, b_{2} \in \mathbb{R}$. With the complex numbers $d:=d_{1}+i d_{2}, e:=\left(a_{1}+i a_{2}\right) / 2$ and $f:=\left(b_{1}+i b_{2}\right) / 2$ in $\mathbb{C}$ this yields

$$
\beta: z \longrightarrow \beta(z)=d+z(e+f)+\bar{z}(e-f) .
$$

We write $\beta\left(Q_{k}\right):=\left(\beta\left(q_{0, k}\right), \ldots, \beta\left(q_{n-1, k}\right)\right)^{t}$. The affine mapping $\beta$ is regular for $a_{1} b_{1}+a_{2} b_{2} \neq 0$ or, equivalently, $e \bar{f}+\bar{e} f \neq 0$. 


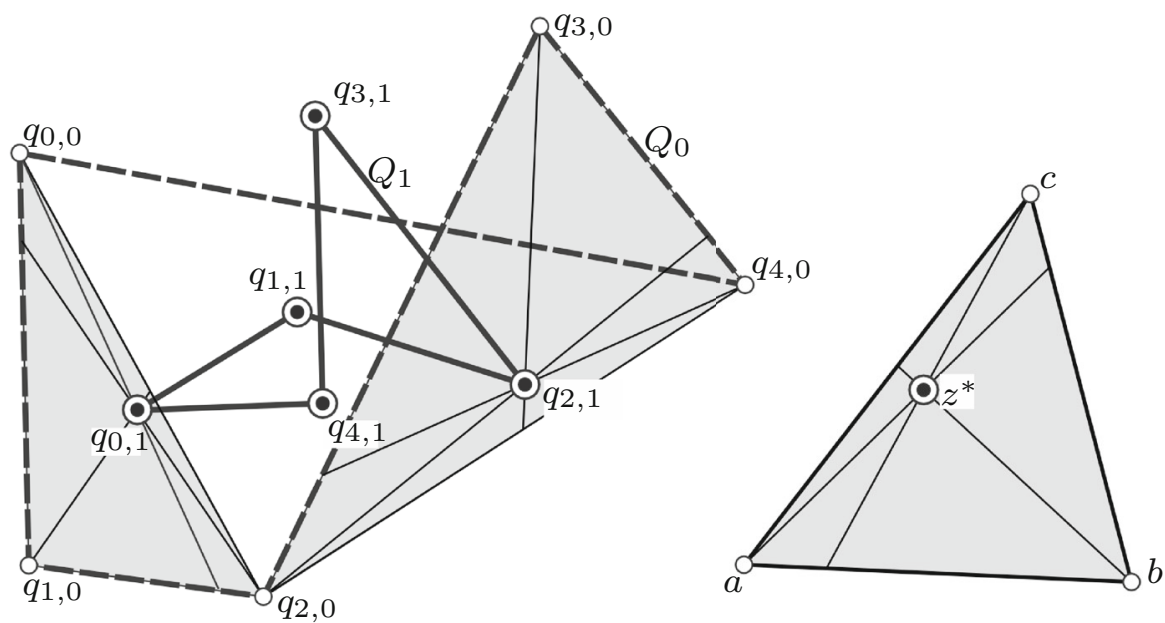

Fig. 1 The case $n=5$ : the polygon $Q_{0}$ and the first generation polygon $Q_{1}$ for $z^{*}=u a+v b+w c$

\section{The iteration process and regular $\boldsymbol{n}$-gons}

Formula (1) can formally be written as

$$
\begin{gathered}
\left(\begin{array}{c}
q_{0, k} \\
\vdots \\
q_{n-1, k}
\end{array}\right)=\left(\begin{array}{cccccc}
u & v & w & 0 & \ldots & 0 \\
0 & u & v & w & \ldots & 0 \\
\vdots & \vdots & \ddots & \ddots & \ddots & \vdots \\
0 & \ldots & u & v & w \\
w & 0 & \ldots & \ldots & u & v \\
v & w & 0 & \ldots & 0 & u
\end{array}\right)\left(\begin{array}{c}
q_{0, k-1} \\
\vdots \\
q_{n-1, k-1}
\end{array}\right) \Leftrightarrow \\
Q_{k}=M \cdot Q_{k-1}
\end{gathered}
$$

with the $n \times n$ - matrix $M \in \mathbb{R}^{n, n}$. As $M$ is a so-called circulant matrix, discrete Fourier transformations and the $n$th roots of unity $\zeta_{j}:=\exp \left(i \frac{2 j \pi}{n}\right)=\cos \frac{2 j \pi}{n}+i \sin \frac{2 j \pi}{n}$ $(j \in \mathbb{Z}$ ) play a pivotal role for us (see Pech 2002, Schoenberg 1950 and Ziv 2002). For them we have $\zeta_{j}=\zeta_{j \bmod n}, \bar{\zeta}_{j}=\zeta_{n-j}(j \in \mathbb{Z})$. We define the vectors

$$
P_{j}:=\left(\zeta_{j}^{0}, \ldots, \zeta_{j}^{n-1}\right)^{t} \quad j \in \mathbb{Z}
$$

We have $P_{n-j}^{t} M=\left(u \zeta_{j}^{0}+v \zeta_{j}^{1}+w \zeta_{j}^{2}\right) P_{n-j}^{t}$ and $M P_{j}=\left(u \zeta_{j}^{0}+v \zeta_{j}^{1}+w \zeta_{j}^{2}\right) P_{j}$ for all $j \in \mathbb{J}:=\{0, \ldots, n-1\}$. Therefore the vectors $P_{n-j}^{t}$ and $P_{j}$ are left and right eigenvectors of the matrix $M$ to the same eigenvalue

$$
\lambda_{j}:=u \zeta_{j}^{0}+v \zeta_{j}^{1}+w \zeta_{j}^{2}(j \in \mathbb{J})
$$

Owing to $(u, v, w) \in \mathbb{R}^{3}$ we have $\bar{\lambda}_{j}=\lambda_{n-j}$ for all $j \in \mathbb{J}$. 


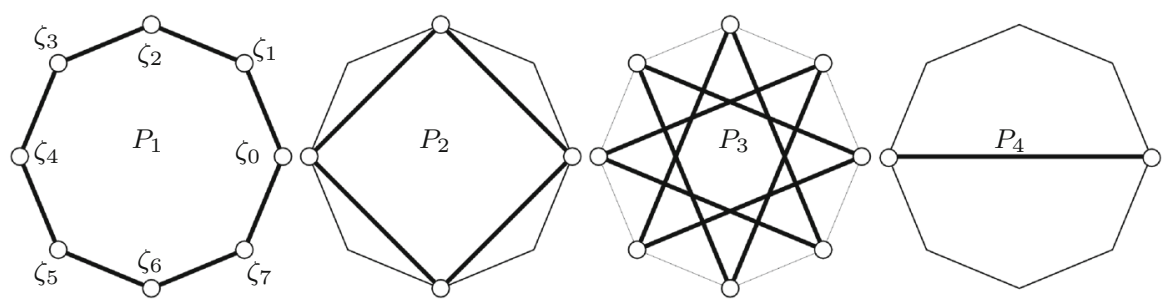

Fig. 2 The case $n=8$ : the regular octogons $P_{1}, \ldots P_{4}$ of first, second, third and forth kind

Remark 3.1 For $j \in \mathbb{J}^{*}:=\{1, \ldots, n-1\}$ the elements of $P_{j}$ can be interpreted as a collection of $n$ points $\zeta_{j}^{k}(k \in \mathbb{J})$ equally distributed on the unit circle. They determine vertices of a so-called 'regular $n$-gon of $j$ th kind'. The regular $n$-gon of $(n-j)$ th kind $P_{n-j}$ is the reflection of $P_{j}$ in the real axis $y=0$ and is affinely equivalent to $P_{j}$. If $j$ and $n$ are relatively prime the polygon $P_{j}$ is either a regular $n$-gon or an $n$-sided regular star. If $j$ is a divisor of $n$ with $n=j m$ the polygon $P_{j}$ either is a regular $m$-gon or a regular star with $m$ vertices on the unit circle which has to be counted $j$ times. For $j \in \mathbb{J}^{*}$ these regular $n$-gons $P_{j}$ of $j$ th kind share the origin 0 as their center of gravity. Figure 2 displays the situation for $n=8$ : The regular octogons $P_{7}, P_{6}, P_{5}$ coincide with $P_{1}, P_{2}, P_{3}$, but have opposite orientation.

\section{The normal form of the matrix representation}

We define two matrices

$$
L:=\frac{1}{\sqrt{n}}\left(P_{n}, P_{n-1}, \ldots, P_{1}\right) \quad \text { and } \quad R:=\frac{1}{\sqrt{n}}\left(P_{0}, P_{1}, \ldots, P_{n-1}\right) .
$$

Both matrices, $L$ and $R$, are symmetric and regular for $n>1$ (see Pech 2002, Schoenberg 1950 and Ziv 2002). Moreover, we have $L=\bar{R}$ and $L \cdot R=I_{n, n}$ with the $n \times n$ unit matrix $I_{n, n}$; the matrices $L$ and $R$ are unitary $n \times n$-matrices in $\mathbb{C}^{n \times n}$. This way we have

$$
L \cdot M \cdot R=D\left(\lambda_{0}, \ldots, \lambda_{n-1}\right)
$$

with the diagonal matrix $D\left(\lambda_{0}, \ldots, \lambda_{n-1}\right)$ containing the eigenvalues $\lambda_{j}$ of $M$ as elements of the main diagonal. We now have

$$
\begin{aligned}
& L \cdot Q_{k}=D\left(\lambda_{0}, \ldots, \lambda_{n-1}\right) \cdot L \cdot Q_{k-1} \text { and } \\
& L \cdot Q_{k}=D\left(\lambda_{0}, \ldots, \lambda_{n-1}\right)^{k} \cdot L \cdot Q_{0}(k \in \mathbb{N}-\{0\})
\end{aligned}
$$

For the $j$ th component of the vectors in (8) this can be written as

$$
\sum_{m=0}^{n-1} \zeta_{n-j}^{n-m} q_{m, k}=\lambda_{j}^{k} \sum_{m=0}^{n-1} \zeta_{n-j}^{n-m} q_{m, 0}=\left(u \zeta_{j}^{0}+v \zeta_{j}^{1}+w \zeta_{j}^{2}\right)^{k} \sum_{m=0}^{n-1} \zeta_{n-j}^{n-m} q_{m, 0}
$$


for all $k \in \mathbb{N}-\{0\}$ and $j \in \mathbb{J}$. The element $j=0$ yields $\lambda_{0}^{k}=(u+v+w)^{k}=1 \forall k \in \mathbb{N}$ and therefore $\sum_{m=0}^{n-1} q_{m, k}=\sum_{m=0}^{n-1} q_{m, 0}$ for all $k \in \mathbb{N}-\{0\}$. Thus, all polygons $Q_{k}$ have the same center of gravity $(k \in \mathbb{N})$. Without loss of generality, we put this center of gravity into the origin 0 of our coordinate system. Thus, we assume that the initial polygon $Q_{0}$ has its center of gravity in 0 :

$$
\frac{1}{n} \sum_{m=0}^{n-1} q_{m, 0}=0
$$

As the matrix $L$ is regular the initial polygon $Q_{0}$ can be explicitly recovered from the vector

$$
B:=L Q_{0} \text { with } B=\left(0, b_{1}, \ldots, b_{n-1}\right)^{t} \in \mathbb{C}^{n-1} .
$$

Under our assumption (10) the first coordinate of the vectors in (8) is 0 . Thus, we do not alter the recursion if we replace the matrix $D\left(\lambda_{0}, \ldots, \lambda_{n-1}\right)$ in (8) by the diagonal matrix $D^{*}:=D\left(0, \lambda_{1}, \ldots, \lambda_{n-1}\right)$. With that and (11) in mind, the iteration process is described by

$$
L \cdot Q_{k}=D^{* k} \cdot B \Leftrightarrow Q_{k}=\frac{1}{\sqrt{n}} \sum_{j \in \mathbb{J}^{*}} \lambda_{j}^{k} b_{j} P_{j} \quad(k \in \mathbb{N}) .
$$

\section{Definition of affine regularization}

Just like the iterations defined in Donisi et al. (2016) and Ziv (2002), iteration (1) also seems to regularize 'in a way' for certain $(u, v, w) \in \mathbb{R}^{3}$ independent of the choice of the initial polgon $Q_{0}$. In order to discuss this interesting property we compare the $n$-gons $Q_{k}$ with a regular prototype $n$-gon $P_{j}$ of kind $j$.

Definition 5.1 We call the procedure (1) affinely regularizing of kind $j \in \mathbb{J}^{*}$ if, for general input data (initial polygon $Q_{0}$ ), there exist affine mappings $\beta_{k}$ transforming $Q_{k}=\left(q_{0, k}, \ldots, q_{n-1, k}\right)^{t}$ into polygons $\beta_{k}\left(Q_{k}\right)$ with the property that for all $k \in \mathbb{N}$ the series $\Delta_{k}$ of sums of the squared distances of the points of $\beta_{k}\left(Q_{k}\right)$ to the regular prototype polygon $P_{j}(4)$ of kind $j$ is a null series.

Thus, the iterative processes (1) is affinely regularizing of kind $j$ if there exist affine mappings $\beta_{k}$ with:

$$
\Delta_{k}:=\sum_{m=0}^{n-1} \operatorname{dist}^{2}\left(\beta_{k}\left(q_{m, k}\right), \zeta_{j}^{m}\right)=\left(P_{j}-\beta_{k}\left(Q_{k}\right)\right)^{t} \cdot\left(\overline{P_{j}-\beta_{k}\left(Q_{k}\right)}\right)
$$

has the property $\lim _{k \rightarrow \infty} \Delta_{k}=0$.

Remark 5.2 - It is important to note that definition 5.1 does not require the existence of a limit polygon $Q_{k}$ for $k \longrightarrow \infty$. In general for the iterative processes (1) such a limit polygon will not exist. 'Affinely regularizing' in our sense is a property of the affine shape of the series $Q_{k}$ alone. 
- In order to be able to measure the sum of the squared distances in Definiton 5.1 we need the Euclidean structure of the plane.

- Our definiton makes use of affine mappings $\beta_{k}$ transforming $Q_{k}$ 'close to some regular prototype polygon' $P_{j}$ of fixed size. In order to define the regulization of the iteration process it would not be enough to do it the other way round: the existence of affine mappings $\gamma_{k}$ transforming the prototype polygon $P_{j}$ close to the polygons $Q_{k}$ (again with sums of squared distances forming a null series) falls short of our requirements. The following counterexample demonstrates that fact: Given a series of polygons $Q_{k}:=(1 / 2)^{k} Q_{0}$ that are homothetic copies of an initial polygon $Q_{0}$. For any $j \in \mathbb{J}^{*}$ the affine mappings $\gamma_{k}\left(P_{j}\right):=(1 / 2)^{k} P_{j}$ yield sums of squared distances of corresponding vertices of $Q_{k}$ and $\gamma_{k}\left(P_{j}\right)$ forming a null series independent from the input data $Q_{0}$. As $Q_{k}$ is homothetic to a polygon $Q_{0}$ 'of general shape' this process, in general, is far from being affinely regularizing.

\section{The affine shape of $Q_{k}$}

The shape of the polygons $Q_{k}$ depends on the input data set $Q_{0}$ and the barycentric corordinates $(u, v, w)$ of the point $z$ with $u+v+w=1$. $(u, v, w)$ uniquely determine the diagonal matrix $D^{*}=D\left(0, \lambda_{1}, \ldots, \lambda_{n-1}\right)$. We compute the norms $n_{j}:=\sqrt{\lambda_{j} \lambda_{j}^{-}}$ of the eigenvalues $\lambda_{j}$ for $j \in \mathbb{J}^{*}$ and have

$$
\begin{aligned}
n_{j}^{2} & =\lambda_{j} \bar{\lambda}_{j}=u^{2}+v^{2}+w^{2}+v(u+w)\left(\zeta_{j}+\zeta_{-j}\right)+u w\left(\zeta_{j}^{2}+\zeta_{-j}^{2}\right) \\
& =u^{2}+v^{2}+w^{2}+2 v(u+w) \cos (2 \pi j / n)+2 u w \cos (4 \pi j / n)
\end{aligned}
$$

$\left(j \in \mathbb{J}^{*}\right)$. The spectral radius of the matrix $D^{*}$ is the maximal value $N:=$ $\max \left\{n_{1}, \ldots, n_{n-1}\right\}$ of the norms of the eigenvalues $\lambda_{j}$ for $j \in \mathbb{J}^{*}$. We assume that the barycentrics $(u, v, w)$ with $u+v+w=1$ are chosen generally in the sense that not all $\lambda_{1}, \ldots, \lambda_{n-1}$ vanish.

It is easy to see that the exceptional case $\lambda_{1}=\cdots=\lambda_{n-1}=0$ can only occour in the case where $n=3$ and, additionally, $(u, v, w)=(1 / 3,1 / 3,1 / 3)$. Therefore, this case in general yields iterated series of 'degenerate triangles' $Q_{k}$ that are formed by the center of gravity 0 . This trivial case shall be excluded from now on by putting $n>3$.

In all other cases we have that $N>0$. So we can define the two sets of indices

$$
\mathbb{J}_{1}:=\left\{j \in \mathbb{J}^{*} /\left|\lambda_{j}\right|=N\right\} \neq \varnothing \text { and } \mathbb{J}_{2}:=\mathbb{J}^{*}-\mathbb{J}_{1} \text {. }
$$

Note that, according to (5), for any $j^{*} \in \mathbb{J}_{1}$ the index $n-j^{*}$ is also contained in $\mathbb{J}_{1}$ (only for even $n$ and $j^{*}=n / 2$ these two indices are coincident) and we have

$$
\frac{\left|\lambda_{j}\right|}{N}=1 \forall j \in \mathbb{J}_{1}, \quad \frac{\left|\lambda_{j}\right|}{N}<1 \forall j \in \mathbb{J}_{2} .
$$

Figure 3 shows the positions of $\lambda_{j}$ for $n=8$ and the barycentrics $(u, v, w)$ of $z^{*}$ with respect to the triangle $a b c$. For this special case we have $\mathbb{J}_{1}=\{1,7\}$. 

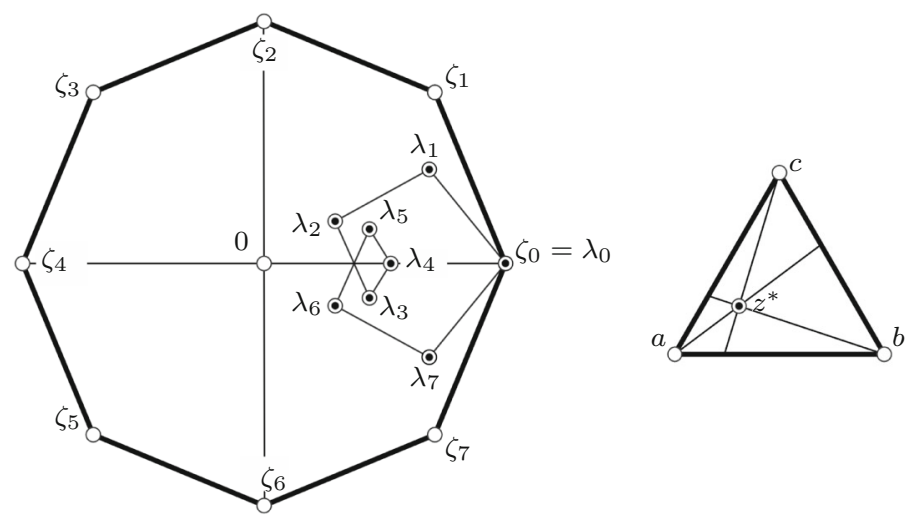

Fig. 3 The case $n=8$ : the eigenvalues $\lambda_{j}$ for $n=8$ and $z^{*}=u a+v b+w c$

Equation (12) yields

$$
Q_{k}=\frac{N^{k}}{\sqrt{n}}\left(\sum_{j \in \mathbb{J}_{1}}\left(\frac{\lambda_{j}}{N}\right)^{k} b_{j} P_{j}+\sum_{j \in \mathbb{J}_{2}}\left(\frac{\lambda_{j}}{N}\right)^{k} b_{j} P_{j}\right) .
$$

Independent of the input data $b_{j}$ the coefficients $\left(\frac{\lambda_{j}}{N}\right)^{k} b_{j}$ of $P_{j}$ in the second sum in (17) form null series for all $j \in \mathbb{J}_{2}$ and $k \longrightarrow \infty$. The coefficients in the first sum are finite complex numbers for all $k \in \mathbb{N}$. Depending on the spectral radius $N$ formula (17) delivers three possibilities:

- For $N<1$ the series $Q_{k}$ decreases for increasing $k$ and tends towards the center of gravity 0 .

- For $N=1$ the series $Q_{k}$ remains finite, but in general changes its position for different $k$.

- For $N>1$ the series $Q_{k}$ increases for increasing $k$ and seems to explode.

For all these possibilities, though, the series $Q_{k}$ seems to be affinely regularizing. This is to be discussed in three cases:

Case $A$ The index set $\mathbb{J}_{1}$ contains exactly one element.

As stated before, this is only possible for even $n$ and special barycentrics $(u, v, w)$ such that $\mathbb{J}_{1}=\{n / 2\}, \mathbb{J}_{2}=\mathbb{J}^{*}-\{n / 2\}$. We have $\zeta_{n / 2}=-1$, and therefore $\lambda_{n / 2}=$ $u-v+w \in \mathbb{R}$. This yields $\left|\lambda_{n / 2}\right| / N=(-1)^{s}$ with $s \in\{0,1\}$ depending on the sign of $u-v+w$. The only element in the first sum of (17) has the coefficient $c_{k}^{*}:=(-1)^{s k} b_{n / 2}$. For a general input polygon $Q_{0}$ we have $b_{n / 2} \neq 0$ and therefore $c_{k}^{*} \neq 0$. We can define the following affine image of the $k$ th generation $Q_{k}$ :

$$
\beta_{k}\left(Q_{k}\right):=\frac{\sqrt{n}}{c_{k}^{*} N^{k}} Q_{k}=P_{n / 2}+\frac{1}{c_{k}^{*}} \sum_{j \in \mathbb{J}_{2}}\left(\frac{\lambda_{j}}{N}\right)^{k} b_{j} P_{j} .
$$


With (17) we compute the sum of squared distances of $\beta_{k}\left(Q_{k}\right)$ from $P_{n / 2}$ via

$$
\Delta_{k}=n\left(\sum_{j \in \mathbb{J}_{2}}\left(\frac{\bar{\lambda}_{j} \lambda_{j}}{N^{2}}\right)^{k} \bar{b}_{j} b_{j}\right) /\left(\bar{b}_{n / 2} b_{n / 2}\right)
$$

As stated before, for all $j \in \mathbb{J}_{2}$ the value $\left(\frac{\bar{\lambda}_{j} \lambda_{j}}{N^{2}}\right)^{k}$ decreases for increasing $k$ and $\lim _{k \longrightarrow \infty}\left(\frac{\bar{\lambda}_{j} \lambda_{j}}{N^{2}}\right)^{k}=0$. Thus, according to our Definition 5.1 the iteration process in Case A for general input data is affinely regularizing of kind $n / 2$. For large $k$ generation $Q_{k}$ will approach the shape of the degenerate regular $n$-gon $P_{n / 2}$ : It consists of two points, each of them counted $n / 2$ times.

Case $B$ The index set $\mathbb{J}_{1}$ contains exactly two different elements: $\mathbb{J}_{1}=\left\{j^{*}, n-j^{*}\right\}$ with $j^{*} \neq n / 2$ (in Fig. 3 we have an example for $n=8$ with $j^{*}=1$ ). We put $\lambda_{j^{*}}=N e^{i \phi}$ and $\lambda_{n-j^{*}}=N e^{-i \phi}$ with some real angle $\phi \in[0,2 \pi)$. Then (17) yields

$$
Q_{k}=\frac{N^{k}}{\sqrt{n}}\left(e^{i k \phi} b_{j^{*}} P_{j^{*}}+e^{-i k \phi} b_{n-j^{*}} \bar{P}_{j^{*}}+\sum_{j \in \mathbb{J}_{2}}\left(\frac{\lambda_{j}}{N}\right)^{k} b_{j} P_{j}\right)
$$

We define $\sigma:=b_{j^{*}} \bar{b}_{j^{*}}-b_{n-j^{*}} \bar{b}_{n-j^{*}}(\sigma \in \mathbb{R})$. For general input data we have $\sigma \neq 0$ and we can define affine images of the $k$ th generation $Q_{k}$ :

$$
\begin{aligned}
\beta_{k}\left(Q_{k}\right) & :=\frac{\sqrt{n}}{\sigma N^{k}} e^{-i k \phi}\left(\bar{b}_{j^{*}} Q_{k}-b_{n-j^{*}} \bar{Q}_{k}\right) \\
& =P_{j^{*}}+\frac{1}{\sigma} e^{-i k \phi} \sum_{j \in \mathbb{J}_{2}}\left(\frac{\lambda_{j}}{N}\right)^{k}\left(b_{j} \bar{b}_{j^{*}}-\bar{b}_{n-j} b_{n-j^{*}}\right) P_{j}
\end{aligned}
$$

Now we compute the sum $\Delta_{k}:=\left(P_{j^{*}}-\beta_{k}\left(Q_{k}\right)\right)^{t} \cdot \overline{\left(P_{j^{*}}-\beta_{k}\left(Q_{k}\right)\right)}$ of squared distances of respective points of $\beta_{k}\left(Q_{k}\right)$ and $P_{j^{*}}$. Short computation yields

$$
\Delta_{k}=\frac{n}{\sigma^{2}} \sum_{j \in \mathbb{J}_{2}}\left(\frac{\bar{\lambda}_{j} \lambda_{j}}{N^{2}}\right)^{k}\left(b_{j} \bar{b}_{j^{*}}-\bar{b}_{n-j} b_{n-j^{*}}\right)\left(\bar{b}_{j} b_{j^{*}}-b_{n-j} \bar{b}_{n-j^{*}}\right)
$$

The only factors in (22) depending on $k$ are $\left(\frac{\bar{\lambda}_{j} \lambda_{j}}{N^{2}}\right)^{k}$. For each $j \in \mathbb{J}_{2}$ these terms form null series for $k \in \mathbb{N}$. The finite sum $\Delta_{k}$ of null series delivers a null series again and we have $\lim _{k \rightarrow \infty} \Delta_{k}=0$. Thus, the corresponding iteration process is affinely regularizing of kind $j^{*}$.

Case $C$ The index set $\mathbb{J}_{1}$ contains more than two different elements $j^{*}, j^{* *}, n-j^{*}$ with $j^{* *} \neq j^{*}$ and $j^{* *} \neq n-j^{*}$. According to (14) we have

$$
\begin{aligned}
& v(u+w)\left(\zeta_{j^{*}}+\zeta_{-j^{*}}-\zeta_{j^{* *}}-\zeta_{-j^{* *}}\right) \\
& \quad+u w\left(\zeta_{j^{*}}^{2}+\zeta_{-j^{*}}^{2}-\zeta_{j^{* *}}^{2}-\zeta_{-j^{* *}}^{2}\right)=0 .
\end{aligned}
$$


In general this is the equation of a conic section in the plane of the triangle $a b c$. For these special choices of the barycentrics $(u, v, w)$ we cannnot give any affine mappings like in Cases $\mathrm{A}$ and $\mathrm{B}$ for general input polygon $Q_{0}$ which deliver a null series for the corresponding distance functions.

We have:

Theorem 6.1 For a general input polygon $Q_{0}$ and general barycentrics $(u, v, w)$ the iteration process (1) is affinely regularizing.

Remark 6.2 In general, the shape of $Q_{k}$ gradually approaches that of an affinely transformed regular $n$-gon $P_{j^{*}}$ of kind $j^{*}$. The kind $j^{*}$ of this affine regularization belongs to those indices $j^{*}$ which determine the spectral radius $N=\left|\lambda_{j^{*}}\right|$ of the transformation matrix $D^{*}=D\left(0, \lambda_{1}, \ldots, \lambda_{n-1}\right)$.

The exceptional cases are addressed in

Theorem 6.3 In the following exceptional cases the iteration process (1), in general, fails to regularize:

- Case A We have even $n$, special barycentrics $(u, v, w)$ and get case $A$ with $c_{k}^{*}=0$. The initial polygon $Q_{0}$ has a special shape with $b_{n / 2}=0$.

- Case $B$ The input polygon $Q_{0}$ has a special shape with $\sigma:=b_{j} \bar{b}_{j^{*}}-$ $b_{n-j^{*}} \bar{b}_{n-j^{*}}=0$.

- Case C The input barycentrics $(u, v, w) \in \mathbb{R}^{3}$ with $u+v+w=1$ comply with (23) for suitable triples $\left(j^{*}, j^{* *}, n-j^{*}\right)$ consisting of three different indices from the index set $\mathbb{J}_{1}$.

Remark 6.4 As long as we are not in the realm of the exceptional cases listed in Theorem 6.3 the regularizing property of Theorem 6.1 remains valid, even if some of the data points of the initial polygon $Q_{0}$ or of some further generation are coincident.

Figure 4 shows an example for $n=7$ with the barycentrics $(u, v, w)=$ $(0.5,-0.2,0.7)$ of $z^{*}$. We get $\left(n_{1}^{2}, n_{2}^{2}, n_{3}^{2}\right) \approx(0.57,0.51,1.28)$ and therefore we have $\mathbb{J}_{1}=\{3,4\}$. This way we have an example for case $B$. The figure shows $Q_{0}$ and the 10th generation $Q_{10}$ which approaches the affine image of the regular star 7-gon $P_{3}$.

\section{Generalisations}

The considerations from above can be modified to obtain further affinely regularizing iteration processes. Instead of the planar affine mappings used in (1) we can also use affine mappings from an $n$-dimensional affine space with basic points in the vertices of a simplex $a_{0}, \ldots, a_{n-1}$. A point $z^{*}:=\sum_{\mu=0}^{n-1} u_{\mu} a_{\mu}$ with barycentrics $\left(u_{0}, \ldots, u_{n-1}\right)$ and $\sum_{\mu=0}^{n-1} u_{\mu}=1$ with respect to that simplex then is mapped to the plane of the initial polygon $Q_{0}$ via affine mappings 


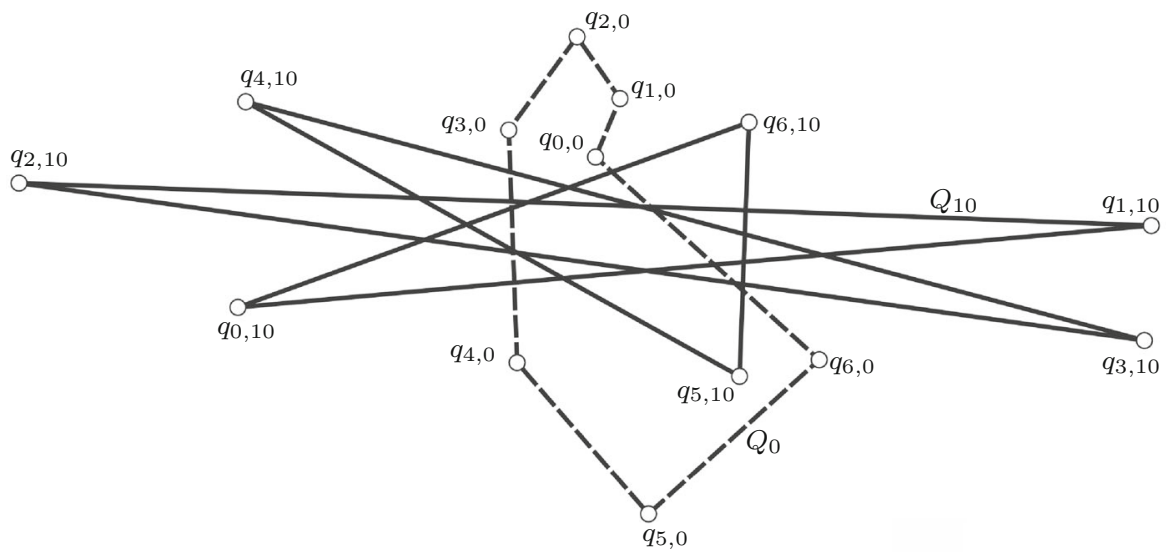

Fig. 4 The case $n=7$ with $\mathbb{J}_{1}=\{3,4\}$

$$
\begin{aligned}
\alpha_{j, k}\left(z^{*}\right): z^{*} & =\sum_{\mu=0}^{n-1} u_{\mu} a_{\mu} \longrightarrow q_{j, k} \text { with } \\
q_{j, k} & :=\sum_{\mu=0}^{n-1} u_{\mu} q_{j+\mu(\bmod n), k-1}(j \in \mathbb{J} ; k \in \mathbb{N}-\{0\}) .
\end{aligned}
$$

Such an iteration process has been studied by Schoenberg (1950). The author did not state results on the affine shape of $Q_{k}$. In this case the eigenvalues $\lambda_{j}$ are given by

$$
\lambda_{j}:=\sum_{\mu=0}^{n-1} u_{\mu} \zeta_{j}^{\mu}(j \in \mathbb{J})
$$

These generalized iteration processes show the same behavior as that described in Theorems 6.1 and 6.3. Again, for general input data $Q_{0}$ and general $u_{\mu}(\mu=0, \ldots, n-1)$ with $\sum_{\mu=0}^{n-1} u_{\mu}=1$ the corresponding iteration processes are still affinely regularizing. Our results could be viewed as a nice add-on to I. J. Schoenberg's paper (Schoenberg 1950).

Acknowledgements Open access funding provided by Graz University of Technology.

Open Access This article is distributed under the terms of the Creative Commons Attribution 4.0 International License (http://creativecommons.org/licenses/by/4.0/), which permits unrestricted use, distribution, and reproduction in any medium, provided you give appropriate credit to the original author(s) and the source, provide a link to the Creative Commons license, and indicate if changes were made.

\section{References}

Donisi, S., Martini, H., Vincenzi, G., Vitale, G.: Polygons derived from polygons via iterated constructions. Electron. J. Differ. Geom. Dyn. Syst. 18, 14-31 (2016) 
Pech, P.: The harmonic analysis of polygons and Napoleon's theorem. J. Geom. Gr. 5(1), 13-22 (2001)

Radcliffe, D.: Inscribed polygons and the Fourier transform. https://mathblag.wordpress.com/2013/10/08/ inscribed-polygons-and-the-fourier-transform/ (2016)

Schoenberg, I.J.: The finite Fourier series and elemenatry geometry. Am. Math. Monthly 57, 390-404 (1950)

Ziv, B.: Napoleon-like configurations and sequences of triangles. Forum Geom. 2, 115-128 (2002) 